\title{
Fuzzy Utilization in Speech Recognition and its Different Application
}

\author{
Bennilo Fernandes. J, Kasi Prasad Mannepalli , Agilesh Saravanan, KTPS Kumar
}

\begin{abstract}
Talk affirmation is one among the basic zones in cutting edge talk process. The examination of talk affirmation may be a bit of an examination for "artificial intelligence" machines that may "hear" and "appreciate" the verbally communicated data. The customary ways for talk affirmation like HMM and DTW, are outrageously inconvenient and time excellent. As such formal Fuzzy justification may be an endeavor in cutting edge talk process for the convincing portrayal of talk affirmation in a couple of utilization. The approach masterminded in the midst of this paper streamlines the utilization of fuzzy in talk affirmation and make the data dealing with time shorter. The case considered in the midst of this paper is that the least mind boggling, i.e., the example of speaker dependence, little vocabulary and disconnected words. There are various spectral and common choices isolated from human talk. The present ways for tendency acknowledgment from voice use basically MFCC and Energy feature. This paper briefs an overview concerning the present work on talk feeling ID strong for completing more examination by feathery approach.
\end{abstract}

Keywords-Emotional Speech Recognition, Fuzzy, HMM, NN, , Applications.

\section{INTRODUCTION}

The talk sign is that the speediest and besides the most widely recognized technique of correspondence between individuals. This reality has pushed researchers to think about talk as an expedient and efficient strategy of collaboration among human and machine. In any case, this needs the machine ought to have the sufficient knowledge to perceive human voices. Since the late fifties, there has been tremendous examination on talk affirmation, that suggests the procedure for changing the human talk into a game plan of words. In any case, paying little heed to the charming headway made in talk affirmation, we will in general are still off from having a trademark relationship among man and machine as a result of the machine doesn't see the sentiment of the speaker. This has displayed a comparably continuous examination field, particularly talk eager affirmation, that is defined as removing the sentiment of a speaker from his or her talk. It's believed that enthusiastic talk affirmation may be wont to separate pleasing phonetics from talk, and thus, improves the display of talk affirmation systems.

Revised Version Manuscript Received on Jun 20, 2019.

J.Bennilo Fernandes, Asst.Professor, Department of Electronics and Communication Engineering, Koneru Lakshmaiah Education Foundation, Guntur, Andhra Pradesh.

Kasiprasad Mannepalli, Assoc. Professor, Department of Electronics and Communication Engineering, Koneru Lakshmaiah Education Foundation ( K L University, Vijayawada), Andhra Pradesh.

R. Agilesh Saravanan, Asst. Professor, Department of Electronics and Communication Engineering, Koneru Lakshmaiah Education Foundation ( K L University, Vijayawada), Andhra Pradesh.

K.T.P.S Kumar, Asst. Professor, Department of Electronics and Communication Engineering, Koneru Lakshmaiah Education Foundation ( K L University, Vijayawada), Andhra Pradesh.

\section{Emotional SPEECH RECOGNition}

Speech enthusiastic affirmation is especially valuable for applications that need trademark man-machine collaboration like net films and pc instructional exercise applications wherever the response of these systems to the customer depends upon the recognized inclination. It's conjointly helpful for in-vehicle board structure wherever data of the psychological state of the reason power is moreover given to the system to begin his/her prosperity. It may be furthermore used as a decisive instrument for counselors. It will be conjointly helpful in customized elucidation structures in the midst of which the sentiment of the speaker expect a vital activity in correspondence between social occasions. In strength cockpits, it's been found that talk affirmation systems arranged to concentrated on talk come through higher execution than those readied by standard talk. Talk feeling affirmation has conjointly been utilized in call center applications and compact correspondence. the most focus of using talk feeling affirmation is to alter the structure response upon acknowledgment disillusionment or unsettling influence inside the speaker's voice Fig 1.

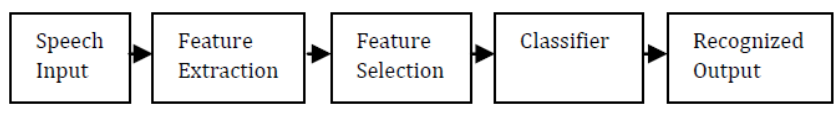

Fig 1. Emotional speech processing procedure.

The errand of talk energetic affirmation is incredibly hard for the resulting reasons. To begin with, it's questionable that talk choices are most overwhelming specifically between sentiments. The acoustic irregularity introduced by the nearness of various sentences, speakers, talking plans, and talking rates incorporates another prevention due to these properties clearly influence most of the ordinary expelled talk choices like pitch, and imperativeness shapes. In addition, there could in like manner be extremely one seen feeling inside the proportional sound-related correspondence; each inclination looks at to an unquestionable piece of the verbally communicated verbalization. besides, it's horribly difficult to work out the breaking points between these parts. Another problematic issue is that at any rate a particular tendency is conveyed generally depends upon the speaker, his or her lifestyle and atmosphere. As a result, it's questionable that feeling the electronic inclination recognizer can distinguish: the long inclination or the transient one. feeling doesn't have a regularly in understanding speculative definition It's been found that the keen system is blended with the sentiments of Joy, Anger, and Fear. 
This incites AN extended heartbeat, higher fundamental sign, changes thorough of processing advancements, greater sub-glottal weight, status of the mouth, and coincidental muscle tremor. The accompanying talk is correspondingly rambunctious, smart and enunciated with ground-breaking high-repeat essentialness, the accompanying ordinary pitch, and increasingly broad pitch change. Despite what might be expected hand, with the energy of the parasympathetic structure, like wretchedness, heartbeat and urgent sign reduction and release will grow, manufacturing talk that is moderate, low-pitched, and with beside no high-repeat imperativeness.

\section{FuZZY In EMotional SPEech ReCOGNition}

The multifaceted idea of tongue has caused individuals to apply different sorts of "fragile" handling procedures for its examination. Other than associated math, connectionist and different philosophies, the feathery justification based approach gives another various to reasonable tongue examination. it's consistently seen that few wonders in tongue advance themselves to depictions by fuzzy number juggling, similarly as feathery sets, cushy relations and numerical reason. By technique a numerical method of reasoning structure and deed right benchmarks, we will as a rule desire that inconveniences in examination of talk are every now and again directed.

As to such a degree as reference is focused on, words and their suggestions (the implied articles or their estimations inside the world) are generally in a cushioned relationship. this is consistently fundamental for grounded systems like overpowering robots. In actuality hand, for the likelihood of human language development examination, words themselves are the objects of delineation. it's typical to accept that the language external haziness likely could be brought into language and feathery numerical philosophies are appropriate gadgets in finding issues.

Feathery basis has been with advancement associated with the system of words' suggestions as related with language external wonders. Soft phonetic descriptors are utilized in the board structures, inside which mappings are as often as possible settled between fuzzy etymological terms and physical sums. "Hot", "cold", for instance, will limit marks for soft sets to that temperature readings are much of the time mapped into enlistment degrees. agent method of reasoning guidelines for the board structures will make due with feathery descriptors in each the premises and moreover the consequents to copy human-like inferencing. Another case of soft application is standard language-driven information look. Here the phonetics of words are as often as possible conveyed as cushioned support works without question information interest keys [Medina, Vila]. A language inside soft treatment is found, inside which influence styles of without question words in reports are constrained as cushy sets. Words addressing sentiments are mapped to those cushioned sets. The capability between this case and moreover the previous 2 is that the last controlled language inside fuzzy wonders.

In phonetic examination there has unendingly been tendency to view etymological classes and structures as cushy substances. this is as often as possible successfully reflected inside the psychological component clear phonetics custom. in this custom, models of ordinary classes and lexical historical background are mulled over as soft and point in interest task. inside the Sixties, Bolinger did examination on soft obvious semantics. Corrigan considers normal classes and besides the related issues reflected in fuzzy illustrative phonetics. Continuums in language and "soft" degrees of subjecthood, noisiness, adjectiveness, etc are mulled over by Comrie. Matthew sees syntactic classes as constant or non-discrete. a couple of functionalists consider etymological classes slant and soft.

\section{LITERATURE REVIEW}

Mohammad Savargiv et al (2018). This paper inspects the related challenges and proposes an assistant methodology for masterminding the substance of excited information. The results layout feelingal information that their substance has been organized by methods for foreseen methodology turn out higher execution inside the inclination affirmation. The framework was associated inside the Persian language and moreover the Persian which means free Text Dataset was imagined. Soft lead of eager states not only inferable in each face and voice, at any rate conjointly it's discernable from the issue content. In spite of the way that the precision is high the excited state like fear, weariness and bewilderment is still in low affirmation rate and enthusiastic state of lively and fair-minded is high in this reasoning. The general precision of energetic state affirmation is around $85 \%$.

Akhmedova, et al (2018). Differential Evolution and Particle Swarm progression (DE+PSO) and created for finding certifiable regarded improvement issues, were associated with the masterminding of fake neural frameworks. Tests showed that each accommodating upgrade methods display world class and consistency inspite of the multifaceted design of the lit up streamlining issues. ANN+COBRA is more lamentable than $\mathrm{ANN}+(\mathrm{DE}+\mathrm{PSO})$, almost certainly accordingly 83 rate and $\mathrm{DE}+\mathrm{PSO}$ rule procured 86 dimension of precision, it's plausible to tutor ANN additional down to earth than ANN+COBRA, as a result of elapid twist solely has PSOlike parts.

Asemi,et al (2018). Here, the adjustive neuro-feathery reasonable thinking system (ANFIS) was used for ASR execution examination in the midst of which it applies an ANN to survey the logical method of reasoning enlistment perform parameters of the fuzzy insightful thinking structure (FIS). The assessment results given in the midst of this paper show the sufficiency of the made strategy. An adjustive neuro-cushioned reasonable thinking structure (ANFIS) to house examination of ASR as a MCDM technique. The ASR impact of MVML-based ASR is 0.74 that is over the impact of MVSL-based ASR. The MVML has the higher show all things considered estimations beside info plentifulness. The MVML oversees botch managing, precision, customer examination and affirmation rate which will be the nice choice as differentiated and the MVSL chiefly based ASR. 
Nancy Bansal, et al (2018). Mel Frequency Cepstral Coefficients has been used for talk feature extraction and in addition to the present formal method of reasoning is likewise used for teaching reason. By then, the upgraded options regards are decreased maltreatment innate algorithmic program. Unquestionably the conspicuousness misuse face and voice produces higher results differentiated and affirmation misuse face only. In base examination the face affirmation supported genetic algorithmic program and in the midst of this examination the major improvement is showed up similar to exactness of the result on same case of pictures and talk. The results exhibit that the foreseen biometric system results in higher security as for 88 rate than the transcendent unimodal distinctive confirmation structures.

Win Chit, et al (2018). This paper proposes the timespace options and repeat territory decisions supported feathery information for consistent talk division task by methods for a nonlinear talk examination. 3 necessary sets to be explicit: low, focus, high at any rate in addition to have out an undefined influence using a ton of fuzzy measures.Examinations are performed on Asian country talk dataset and the results are shown that the masterminded structure are practiced the higher presentations. At any rate this has a couple of limitations because the high common expense of the soft sets and fuzzy rules age.

SemiyeDemircan, et al (2018). Feeling affirmation from talk sign was performed by misuse the soft $\mathrm{C}$-infers rule. The spooky decisions used were Mel repeat cepstral coefficients and straight desire coefficients are associated math decisions were isolated from the supernatural options got inside the examination. Coordinated classifiers like ANN, NB, kNN, and SVM were used for portrayal. The results showed that misuse FCM for preprocessing point exaggerated the accomplishment rate. The examination of the request ways showed that the most outrageous accomplishment rate was gotten as $92.86 \%$ misuse the SVM classifier.

Fan, et a (2018)l. With different AI instruments have a possible to be used for creating perfect operational components for the clearing shapes in light of the versatility to go hunting down the general optima. ANN-GA and ANNPSO models have starting late been made for showing and improvement of the waste clearing frames. Uniform style (UD) together with ANNs are routinely used for envisioning the power of the toxins departure shapes in research offices. Significant learning models, (for instance, convolutional neural framework and DBN) are consistently learn gigantic volumes of data in light of their learning points of confinement are as often as possible compelled by moved their significance and broadness. Thusly, convolutional neural framework and DBN have a possible use for the showing and improvement of the pollutions removal shapes that have enormous and current datasets.

Ghonie, et al (2018). A unique Fuzzy Continuous Speech Recognition approach named FCSR is made courses of action for talk organ pathology conspicuous evidence. new talk weighted territory decisions supported Jacobi-Fourier Moments (JFMs) are given for depiction of voice box pathologies. an organized multi-class soft assistance vector machine (FSVM) model is made to gathering voice box pathologies, wherever package document growth (PIM) agglomeration together with atom swarm improvement (PSO) are used for watchful feathery enlistments and streamlining the conflicts of the piece perform of the FSVM, severally. The examinations license the blend of the new JFWSF and furthermore in light of the fact that the masterminded multi-class FSVM gives $97.6 \%$ of precision.

Khanum, et al (2017). An intriguing affirmation structure that usages Mel Frequency Cepstral Coefficients (MFCC) and Feed Forward Neural Networks (FFNN) for feature extraction and speaker request severally. Soft C Mean pack (FCM) system is likewise used against the removed decisions from the talk, that supports in social occasion inconceivable proportion of data. Subsequently, the system demonstrates higher execution to the extent machine regard and steady distinctive confirmation. In each gathering of speakers, tests were coordinated moved arrangement of neurons inside the covered layer of the neural framework to spot for the best believability. The most flawlessly awesome precision achieved in conspicuous verification of speaker is 98.7\%. Thusly MFCC together with fake neural framework can do higher power similar to regard and time in voice basically based affirmation.

Lazli, et al (2017). A soft innate methodology for talk pack, inside the structure wherever the outcomes of cushy csuggests (FCM) group was used as beginning masses for genetic figurings (GA). The technique is used in a cross breed HMM/ANN structure misuse a designed Neural Network (ANN) to work out the discernment chances inside the states of the Hidden Markov Models (HMM). The results got inside the structure of the ASR show accomplice degree improvement in execution in regard to the 2 basic systems: Hybrid model with k-suggests pack and crossbreed model with FCM.

Yenjerappa, et al (2018). In the space of voice affirmation, sensitive handling method could be a perceived procedure to spot and pack speaker variance's inside the talk signal. In any case at whatever point the sign is tangled by an uproarious sign normal FCM system fails to present the wonderful results. to beat this, Kernel FCM (KFCM) is used in the midst of this paper. PCA helps in lessening the decisions of tangled sign. the omnipresence results are differentiated and remembering that not comparing to the present circumstance PCA abuse KFCM perform and besides the proportionate is given for word affirmation rate. interestingly with FCM and VQ the KFCM performs higher towards the rising of soundness of the talk affirmation structure underneath different vociferous conditions like tangled or included substance disturbances. From the reenactment results we have achieved sixtieth of the accuracy for the separated words in typical for the acoustic technique. doubtlessly Gaussian piece limits performs higher underneath different vociferous conditions. The foreseen system will be additional extended for different combinations of bustle sign like vehicle racket, street fuss, pink fuss, etc., for greater learning sets. this could even be pursued for speaker dependent and autonomous learning sets for getting less botch rates. 
Abdelkefi, et al (2017). Here the arrangement are costumed produce accomplice in seeming Intelligent Tutoring System (EITS) enabling understudy to comprehend his instructional reason adaptively, wherever and at whatever point. Things part is directed by Associate in Nursing expert gathering mindful of the tendency affirmation. Believe it or not, the unmistakable verification of the understudy's spirit is performed in endeavor with the time of talk, outward appearances, and changed movements yet in light of the fact that the effect of the M-learning condition. The tendency to explore and portray the chief standard examinations concerning feeling rule in a couple of setting for Intelligent Tutoring System and learning condition. Thusly foreseen a new out of the crate new structure of Fuzzy Emotional Regulation for ITS, passed on underneath the Multiagent approach.

Akil, et al (2017). The purpose of this examination is to style accomplice degree use of the talk affirmation structure to manage the speed of a DC motor. The Linear prognosticative cryptography (LPC) technique is used inside the talk affirmation structure, tuned by the accommodative Neuro-Fuzzy unique thought Systems (ANFIS) strategy. Voice tests started from within information structure are $83 \%$ with advancement seen by this methodology.

Y. Dai, et al (2017). Develops a wearable biosensor framework to require a phase additional towards step by step life feeling affirmation. Multimodal bio-signals (electroencephalography, beat, skin temperature and circulatory strain) are recorded by the distinguishing segment center points and transmitted to the remote web information center through a body station to respect the webengaged affirmation subject. A reputation driven uneven soft assistance vector machine (RI-FSVM) portrayal logic is made to scale back the troublesome effects achieved by each inside class creaky precedents and between-class unevenness. The RISVM gathering framework diminishes the unpleasant effects realized by between-class imbalance and inside class creaky guides to help the portrayal execution together with S E, S P, weight unit and exactness. Definitely, this paper achieves the precision of $80.94 \%$ for step by step life feeling affirmation, which is $5.62 \%$ over the normal ways KNN and SVM.

Faiyaz, et al (2017). A widely inclusive and savvy portrayal of the patient's energetic state and unites grouping of style conclusions that are fitting for tendency showing and affirmation inside the setting of a certified life social protection surroundings was made. From the preliminary outcomes gave in the midst of this paper, it had been in contestable that the prepared tendency showing structure may be shockingly helpful once associated in unequivocal veritable settings, which a system made by abuse the foreseen framework is in a circumstance to watch sufficiently the sentiments of its customers.

Ben Fredj, et al (2017). The Fuzzy kNN (FkNN), another quality $\mathrm{kNN}$ condition is used for limit talk sound affirmation. Mel Frequency Cepstral Coefficients (MFCC) related to their basic essentialness unfaltering were removed from the talk banner as a commitment of the commonness structure. A relationship of a crisp and cushioned $\mathrm{kNN}$ was performed. Examinations show that FkNN condition not only will cause significant affirmation rates, the mean affirmation rates have earned severally $75.93 \%, 78.57 \%$,

$68.55 \%, 86.35 \%, 68.00 \%, 78.02 \%$ and $82.29 \%$ for vowels, affricates, stops, fricatives, nasals, semi-vowels and quiets. Results exhibits a promising strategy, which will accept an imperative occupation for the headway of a talk sound recognizer.

Ghoniem, et al (2017). Another talk options are foreseen for speaker depiction, that connoted as riffle Packet FourDirectional decisions (WPFDF) and with the speaker check, a Fuzzy Hidden Markoff Model was proposed, the part feathery c-infers (KFCM) is extended to figure cushioned interests of HMMs training tests. Thusly, data setback is reduced furthermore as affirmation rate is exaggerated. The foreseen technique accomplished $98.38 \%$ of affirmation rate. In this way, data hardship is controlled and affirmation rate is distorted.

Maryam Iman, et al (2017). A tendency affirmation system from the face pictures is masterminded in the midst of this paper, which may perceive seven sentiments of human, i.e., six basic appearances also to fair. The organized framework uses the GLCM approach for feature extraction and as such the nearest neighbor $(\mathrm{NN})$ for request. The feathery geometer detachment is used. As a result of quality of absence of lucidity and weakness inside, the fuzzy live is concerned inside the $\mathrm{NN}$ classifier to perceive the sentiments of faces with additional precision. The examinations show the wonderful quality of the familiar affirmation technique differentiated and another component extraction and facial tendency affirmation ways. The masterminded framework is appeared differently in relation to Dennis Gabor and morphological options inside comparative conditions. The examinations exhibited the unrivaled introduction of GLCM.

Lilia and Boukadoum, et al (2017). The examination of preliminary outcomes in cross breed structure abuse Hidden Markov Models/Multi-Layer Perceptron (HMM/MLP) model as acoustic model and supported the Fuzzy C-Means (FCM) pack with streamlining with Genetic algorithmic rule (GA). Show of reasonability of the organized pack approach in colossal vocabulary speaker-self-sufficient constant talk affirmation in regards to the 3 standard structures : Distinct HMM, cross breed HMM/MLP with KMeans and FCM gathering.

Yanti Liliana, et al (2017). The face centers exhibits the dimension of cushioned gathering on eight face emotions, unequivocally shock, disdain, disgust, cheery, stun, sharpness, fear, and unprejudiced. The foreseen ways are supported Active Look Model (AAM) and semi-coordinated Fuzzy C-infers (FCM). we tend to gave the system a shot phycologist Kanade dataset of face appearance that gave eight classes of human tendency. Our ways gain a mean exactness rate of $80.71 \%$ and outflank the dominating Fuzzy illation System. AAM has set up to be a structure and surface extraction strategy as a result of the facial places of interest are dynamic.

Motamed, et al (2016). This paper presents Associate in Nursing upgraded model of cerebrum feelingal learning (BEL) that unions the adaptable Neuro-Fuzzy reasoning System (ANFIS) and Multilayer Perceptron (MLP) for talk feeling affirmation. To describe the speed of talk feeling signals, the ANFIS yields are given to MLP mastermind. 
Preliminary outcomes show that the foreseen rule consolidates an earth shattering ability to identify a human's talk feeling. The most perfectly awesome rate of affirmation over all emotions has a spot with the foreseen model by $72.5 \%$ in two hundred ages.

Shing-Tai, et al (2016). Accomplice in test mode disintegration is used to disconnect the perfect talks from the talk sign spoiled by characteristic clatter. To overhaul the noticeable quality speed, rather than consistent disguised Markov model (CHMM), unquestionable HMM (DHMM) is used here to diminish the count load all through talk affirmation and cushy vector division(FVQ) is used in the showing of DHMM to improve the talk affirmation rates. The talks are subject to moved natural clatters were astoundingly improved, particularly for low SNR talk. Furthermore, the investigations verifed the masterminded strategy has some higher results than those maltreatment the techniques.

Gautam, et al (2015). Irrefutably, disfluency of talk among youngsters is dissected maintained the understandability assessment by talk and language pathologists, which may be past a sensible uncertainty won and long. The display of markers stays such an extraordinary sum underneath individuals. To beat this limitation, a substitution course of action framework supported Fuzzy Petri Nets (FPN) is foreseen to improve the request precision. FPN produces the most perfect precision with $98.31 \%$, trailed by KNN with $97.80 \%$ SVM with $96.44 \%$, LDA produces $95.75 \%$ and RF with fairly cut down that is at 95.36\%.

Paul Choudhury, et al (2015). Spotlighted the impact of learning parameter of target perform, however realizing Fuzzy Vector division on Text Dependent Speaker Verification underneath constrained information condition and furthermore underneath sensible crying atmosphere. Fuzzy philosophies with their variable cushioned parameters may scale back the degradation. The exploratory results performed on telecom information suggests higher results for learning parameter $\mathrm{m}=1.37$ wherever the most precision of the system accomplishes $94.81 \%$.

\section{A. COMPARISION OF DIFFERENT METHODOLOGIES}

\begin{tabular}{|c|c|c|c|c|}
\hline Author & $\begin{array}{l}\text { Year } \\
\text { of } \\
\text { Pub }\end{array}$ & $\begin{array}{l}\text { Methodo } \\
\text { logy }\end{array}$ & $\begin{array}{l}\text { Accura } \\
\text { cy \% }\end{array}$ & Advantages \\
\hline $\begin{array}{l}\text { Mohammad } \\
\text { Savargiv et al }\end{array}$ & 2018 & & 85 & $\begin{array}{l}\text { Emotional states } \\
\text { inferable in each face } \\
\text { and voice }\end{array}$ \\
\hline $\begin{array}{l}\text { Akhmedova, } \\
\text { et al }\end{array}$ & 2018 & $\begin{array}{l}\mathrm{ANN+(D} \\
\mathrm{E}+\mathrm{PSO})\end{array}$ & 83 & $\begin{array}{l}\text { Emotional State } \\
\text { Classification }\end{array}$ \\
\hline Asemi,et al & 2018 & $\begin{array}{l}\text { ANFIS } \\
\& \\
\text { MVML }\end{array}$ & 75 & $\begin{array}{l}\text { Effective error } \\
\text { handling }\end{array}$ \\
\hline $\begin{array}{l}\text { Nancy } \\
\text { Bansal, et al }\end{array}$ & 2018 & & 88 & $\begin{array}{l}\text { Emotional states } \\
\text { inferable with pictures } \\
\text { and speech. }\end{array}$ \\
\hline $\begin{array}{l}\text { SemiyeDemir } \\
\text { can, et al }\end{array}$ & 2018 & FCM & 92.86 & $\begin{array}{l}\text { Effective than ANN, } \\
\mathrm{NB}, \mathrm{kNN} \text {, and SVM }\end{array}$ \\
\hline Fan, et al. & 2018 & $\begin{array}{l}\text { ANN- } \\
\text { GA and } \\
\text { ANN- } \\
\text { PSO }\end{array}$ & 87 & $\begin{array}{l}\text { Removal of massive } \\
\text { and sophisticated } \\
\text { datasets }\end{array}$ \\
\hline Ghonie, et al & 2018 & JFWSF & 97.6 & Multi-class FSVM \\
\hline $\begin{array}{l}\text { Khanum, et } \\
\text { al }\end{array}$ & 2017 & FCM & 98.7 & $\begin{array}{l}\text { Higher potency of } \\
\text { value and time in voice }\end{array}$ \\
\hline
\end{tabular}

\begin{tabular}{|l|l|l|l|l|}
\hline & & & & recognition. \\
\hline Lazli, et al & 2017 & HFCM & 94 & $\begin{array}{l}\text { Hybrid model with k- } \\
\text { means bunch }\end{array}$ \\
\hline $\begin{array}{l}\text { Yenjerappa, } \\
\text { et al }\end{array}$ & 2018 & KFCM & 84 & $\begin{array}{l}\text { Higher recognition } \\
\text { towards lustiness of } \\
\text { the speech }\end{array}$ \\
\hline Akil, et al & 2017 & ANFIS & 83 & $\begin{array}{l}\text { Associate degree } \\
\text { implementation of the } \\
\text { speech recognition } \\
\text { system }\end{array}$ \\
\hline Y. Dai, et al & 2017 & $\begin{array}{l}\text { RI- } \\
\text { FSVM }\end{array}$ & 80.94 & $\begin{array}{l}\text { Boost the } \\
\text { classification } \\
\text { performance together } \\
\text { with SE, SP, weight } \\
\text { unit and accuracy }\end{array}$ \\
\hline $\begin{array}{l}\text { Ben Fredj, et } \\
\text { al }\end{array}$ & 2016 & FkNN & 85 & $\begin{array}{l}\text { Improved Emotional } \\
\text { State Classification }\end{array}$ \\
\hline $\begin{array}{l}\text { Ghoniem, et } \\
\text { al }\end{array}$ & 2017 & KFCM & 98.38 & $\begin{array}{l}\text { Data loss is reduced } \\
\text { also as recognition rate } \\
\text { is exaggerated }\end{array}$ \\
\hline $\begin{array}{l}\text { Lilia } \\
\text { \&Boukadou } \\
\text { m, et al }\end{array}$ & 2017 & $\begin{array}{l}\text { KMeans } \\
\& \text { FCM } \\
\text { cluster }\end{array}$ & 92 & $\begin{array}{l}\text { More accuracy than } \\
\text { HMM, hybrid } \\
\text { HMM/MLP }\end{array}$ \\
\hline $\begin{array}{l}\text { Motamed, et } \\
\text { al }\end{array}$ & 2017 & $\begin{array}{l}\text { ANFIS } \\
\& \text { MLP }\end{array}$ & 72.5 & $\begin{array}{l}\text { Powerful capability to } \\
\text { spot a human's speech } \\
\text { feeling in 200 epochs. }\end{array}$ \\
\hline Gautam, et al & 2016 & FPN & 95.36 & $\begin{array}{l}\text { Enhancement on } \\
\text { classification accuracy }\end{array}$ \\
\hline $\begin{array}{l}\text { Paul } \\
\text { Choudhury, } \\
\text { et al }\end{array}$ & 2015 & FVD & 94.81 & $\begin{array}{l}\text { High sensible in all } \\
\text { atmosphere condition. }\end{array}$ \\
\hline
\end{tabular}

\section{CONCLUSION}

The usage of PCs and human-machine interfaces is growing in our step by step life. Additionally the rising advances in mobile phones have concerned horribly huge humanmachine interfaces. The forefront advancements in talk affirmation have made this interface to play out additional and even more accurately and are driving the globe. Starting at now joined will use these advances even while driving the vehicle since it gives without hands action. The applications epitomize human-machine association wherever individuals will naturally talk with their PCs so the pcs can see the human emotions and correspondingly pick the work to be done and furthermore once the data is given to the PC, it will pick what ought to be the going with call made. the indistinct factor is appropriate to PDAs in light of the way that the sans hands errand are out there together with tendency affirmation.

A lot of vulnerabilities are as yet present for the best guideline to arrange sentiments. extremely astounding mixes of tendency decisions give unmistakable inclination acknowledgment rate. The investigators are so far bantering for what options sway the predominance of tendency in talk. This paper reviews huge highlights inside the progressing headways and examination of talk feeling affirmation with cushy basis and various strategies for game plan. Stood out from various systems, cushioned reasonable technique diminishes the multifaceted nature and enables sufficient element of accuracy in recognizing the eager state of a person. Among the different systems for cushioned method of reasoning Kernel Fuzzy c-suggests (KFCM) and FCM gives more precision rate of $98 \%$. Along these lines unmistakably fuzzy reason can be utilized suitably for different usage of talk affirmation system and can give logically correct results in various constant applications. 


\section{REFERENCES}

1. Mohammad Savargiv, Mohammad Reza Keyvanpour, Soheila MehrMolaei. "A structural method based on fuzzy approach at roducing emotional data". 6th Iranian Joint Congress on Fuzzy and Intelligent Systems (CFIS), IEEE,12 April 2018.

2. Akhmedova, Shakhnaz \& Stanovov, Vladimir \& Semenkin, Eugene. "Cooperation of Bio-inspired and Evolutionary Algorithms for Neural Network Design". Journal of Siberian Federal University - Mathematics and Physics. May 2018.

3. Asemi, Adeleh \& Salim, Siti Salwah \& Shahamiri, Seyed Reza \& Asemi, Asefeh \& Houshangi, Narjes. "Adaptive Neuro-fuzzy Inference System for Evaluating Dysarthric Automatic Speech Recognition (ASR) Systems: A case study on MVML based ASR. Soft Computing”. 2018 10.1007/s00500-018-3013-4.

4. Nancy Bansal, Amit Verm, Iqbaldeep Kaur, Dolly Sharma. "Multimodal biometrics by fusion for security using genetic algorithm". 4th International Conference on Signal Processing, Computing and Control (ISPCC), IEEE, 25 January 2018.

5. Win Chit, Yin \& Khaing, Soe. "Myanmar Continuous Speech Recognition System Using Fuzzy Logic Classification in Speech Segmentation", Association for Computing Machinery, 2018.

6. Semiye Demircan, Humar Kahramanli. "Application of fuzzy C-mean clustering algorithm to spectral features for emotion classification from speech". Neural Computing and Applications, Volume 29, Number 8 , Page 59, 2018

7. Fan, M., Hu, J., Cao, R., Ruan, W., Wei, X., A review on experimental design for pollutants removal in water treatment with the aid of artificial intelligence, Chemosphere (2018), doi: 10.1016/j.chemosphere.2018.02.111.

8. Ghoniem, Ranya \& Shaalan, Khaled. "FCSR - Fuzzy Continuous Speech Recognition Approach for Identifying Laryngeal Pathologies Using New Weighted Spectrum Features". Springer International Publishing AG , 384-395. 10.1007/978-3-319-64861-3_36, 2018.

9. Khanum, Seema \& Abdul, Firos. "A novel speaker identification system using feed forward neural networks", IEEE, 2017 3045-3047. 10.1109/ICECDS.2017.8390014

10. Lazli, Lilia \& Laskri, Mohamed Tayeb \& Boudour, Rachid. "Discriminant learning for hybrid HMM/MLP speech recognition system using a fuzzy genetic clustering". Intelligent Systems Conference, IEEE, 2017, 76-81. 10.1109/IntelliSys.2017.8324351.

11. Yenjerappa, Vani \& A. Anusuya, M. "Noisy Speech Recognition Using Kernel Fuzzy C Means". Springer Nature Singapore Pte Ltd. 2018, 10.1007/978-981-10-9059-2_29

12. Abdelkefi, Mouna \& Kallel, Ilhem. "Towards a fuzzy multiagent tutoring system for M-learners' emotion regulation”. 2017 IEEE, 1-6. 10.1109/ITHET.2017.8067821

13. Akil, Muhammad \& Nurtanio, Ingrid \& Samsoe'oed Sadjad, Rhiza. "A DC motor speed control using the LPC-ANFIS speech recognition system". 2017 IEEE. 215-220. 10.1109/QIR.2017.8168484.

14. Y. Dai, X. Wang, P. Zhang, W. Zhang. "Wearable Biosensor Network Enabled Multimodal Daily-life Emotion Recognition Employing Reputation-driven Imbalanced Fuzzy Classification, Measurement (2017), doi: http://dx.doi.org/10.1016/j.measurement.2017.06.006

15. Faiyaz \& Karyotis, Charalampos \& Iqbal, Rahat \& James, Anne. "An intelligent framework for emotion aware e-healthcare support systems". 2016 IEEE. 1-8. 10.1109/SSCI.2016.7850044.

16. Ben Fredj, Ines \& Ouni, Kais. (2017). "Fuzzy k-Nearest Neighbors applied to phoneme recognition". 2016 IEEE.422-426 10.1109/DT.2017.8012141.

17. Ghoniem, Ranya \& Shaalan, Khaled. "A Novel Arabic Textindependent Speaker Verification System based

18. emotion recognition from face". International Conference on Computer and Knowledge Engineering (ICCKE), IEEE 2017, 10.1109/ICCKE.2017.8167879.

19. Lazli, Lilia \& Boukadoum, Mounir \& Ait Mohamed, Otmane. (2017). "Fuzzy clustering optimized with

20. genetic algorithms: Application for hybrid speech recognition system". 0567-0572. 10.1109/CoDIT.2017.8102654.

21. Yanti Liliana, Dewi \& Widyanto, Rahmat \& Basaruddin, T. "Human emotion recognition based on active appearance model and semisupervised fuzzy C-means". 2016 439-445 10.1109/ICACSIS.2016.7872744.

22. Motamed, Sara \& Setayeshi, Saeed \& Rabiee, Azam. "Speech emotion recognition based on a modified brain emotional learning model". Biologically Inspired Cognitive Architectures. 2017. 19 10.1016/j.bica.2016.12.002.

23. Shing-Tai, PanCheng-Yuan, ChangYi-Heng Tsai. "FPGA-Based Robust Wireless Speech Motion Control for Home Service Robot Subject to
Environmental Noises". International Journal of Fuzzy Systems 2016. DOI: $10.1007 / \mathrm{s} 40815-016-0222-9$

24. Gautam, Sumanlata \& Singh, Latika. (2015). Developmental pattern analysis and age prediction by extracting speech features and applying various classification techniques. International Conference on Computing, Communication and Automation, ICCCA 2015. 83-87. 10.1109/CCAA.2015.7148348.

25. Paul Choudhury, Suman \& Misra, Songhita \& Hussain, Rabul \& Shome, Nirupam \& Kanti Das, Tushar. (2015). Effects of fuzzy parameter on text dependent speaker verification under uncontrolled noisy environment. 10.1109/GCCT.2015.7342703.

26. Mirlab Audio Signal Processing tutorials, "Speech feature MFCC Calculation guide", $\langle$ http://mirlab.org/jang/books/audiosignalprocessing/speechFeatureMfcc .asp> (Browsing Date: 26th February 2016).

27. Elizabeth D. Casserly and David B. Pisoni, 'Speech perception and production'. Wiley Interdiscip Rev Cogn Sci. Author manuscript; available in PMC 2013 Aug 12 http://www.ncbi.nlm.nih.gov/pmc/articles/PMC3740754/, (Browsing Date: 25th October 2015).

28. Anila, R., Revathy, A.: Emotion recognition using continuous density HMM. Communications and Signal Processing (ICCSP), 2015 International Conference on. IEEE (2015)

29. Yogesh, C.K., et al.: Hybrid BBO PSO and higher order spectral features for emotion and stress recognition from natural speech. Appl. Soft Comput. 56, 217-232 (2017)

30. Al-Naser, Mustafa, Elshafei, Moustafa, Al-Sarkhi, Abdelsalam: Artificial neural network application for multiphase flow patterns detection: a new approach. J. Petrol. Sci. Eng. 145, 548-564 (2016)

31. M. Savargiv and A. Bastanfard, "Persian speech emotion recognition",Proceedings of Information and Knowledge Technology (IKT), $20157^{\text {th }}$ Conference on, (2015), 1-5.

\section{AUTHORS PROFILE}

J.Bennilo Fernandes pursuing Ph.D., in KL University under the faculty of Electronics and Communication Engineering, Guntur. He is working as Asst.Professor, Department of Electronics and Communication Engineering, KoneruLakshmaiah Education Foundation, Guntur, Andhra Pradesh. His research interest includes Speech Processing, Image Processing, Embedded Systems and Wireless Sensor Networks. He has published 5 papers in International Journals and 3 Papers in International / National Conferences. He has 3 years of teaching experience.

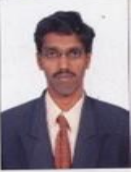

KasiprasadMannepalliis working as Assoc. Professor, Department of Electronics and Communication Engineering, KoneruLakshmaiah Education Foundation ( K L University, Vijayawada), Andhra Pradesh. His research interest includes Speech Processing, Image Processing, machine intelligence. He has published 10 papers in International Journals and 6 Papers in International / National Conferences. He has 12 years of teaching experience

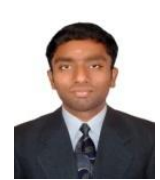

R. Agilesh Saravanan is pursuing Ph.D., degree in K L University, Vijayawada in the faculty of Electronics and Communication Engineering since 2017, Specialized in Signal Processing. He received his Master of Engineering Degree in Applied Electronics from Velammal Engineering College, Anna University,Chennai in 2014 $\mathrm{He}$ received his B.E. Degree in Electronics and Communication Engineering from Chettinad College of Engineering and Technology, Anna University, Chennai in 2012. He has been employed as Assistant Professor in various Engineering College from 2014 till date. His present affiliation is withKoneruLakshmaiah Education Foundation, Guntur, Andhra Pradesh designated as Assistant professor in ECE. His research interest are Ultrasound imaging, Sensor Array Processing and Compressed Sensing

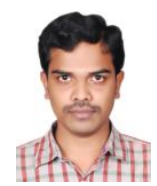

K.T.P.S Kumar, pursuing Ph.D., in KL University under the faculty of Electronics and Communication Engineering, Guntur. He is working as Asst.Professor, Department of Electronics and Communication Engineering, KoneruLakshmaiah Education Foundation, Guntur, Andhra Pradesh. His research interest includes Conformal Antennas, Spiral Antennas, and Embedded Systems. He has published 3 papers in International Journals $\mathrm{He}$ has 11 years of teaching experience. 